\title{
Plenary Sessions
}

\section{Monday, May 13, 2013}

T1:PL

Transcriptional regulation of cellular energy metabolism - with focus on the role of exercise

\section{Pedersen B}

Centre of Inflammation and Metabolism (CIM), Rigshospitalet and University of Copenhagen, Denmark

bkp@rh.dk and http://www.inflammation-metabolism.dk

Recent evidence has identified skeletal muscle as a secretory organ. It has been suggested that cytokines and other peptides that are produced, expressed and released by muscle fibers and exert either autocrine, paracrine or endocrine effects should be classified as "myokines". The muscle secretome consists of several hundred secreted peptides, many of which are transcriptionally regulated by exercise. This finding provides a conceptual basis and a whole new paradigm for understanding how muscles communicate with other organs such as adipose tissue, liver, pancreas, bones and brain. In addition, several myokines exert their effects within the muscle itself. Many proteins produced by skeletal muscle are involved in cellular energy metabolism. Therefore, it is likely that myokines may contribute to mediate some of the health benefits of exercise.

\section{T2:PL}

\section{Energy intake and energy expenditure: The relationship between calorie and body weight}

\section{Schutz Y}

Dept. of Medicine, Integrative Physiology, Univ. of Fribourg, Switzerland

Energy intake (Ein) and energy expenditure (EE) are controlled variables (high CV and flexibility). "Controlling is adapting". They are interrelated with a double feedback system. Body weight (BW) as well as body composition are regulated rather than controlled. "Regulating is homeostasing". The variability of regulated variables is lower than that of controlled variables. For example, despite large day-to-day variation in Ein, BW tends to remain stable over the years in most humans, thanks to compensatory adaptive mechanisms. As a result, no relationship is expected between Ein and BW, since EE (physical activity volume) constitutes a confounding factor. In contrast, the good relationship, in a given individual, between $\mathrm{E}$ balance (Ein minus EE) and BW change has been well demonstrated but it also depends upon the period of time considered and potential methodological errors to assess $\mathrm{E}$ balance. Mathematic modelling in dynamic conditions of BW gain or loss from various input factors (Ein, EE, body composition...) has generated good prediction power in a limited number of individuals (Hall, 2010).

When we consider variation among groups of subjects, recent studies, which imposed a rise in EE consecutive to a fixed imposed exercise stimuli, have highlighted the importance of the large spectrum of BW and body composition responses among subjects (Blundell et al, 2011). This difference is not necessarily due to variations in subjects' compliance levels or in a "compensatory effect" of physical activity.

In conclusion, when looking at the triade relationship among Ein, EE and $\mathrm{BW}$, it is important to consider 2 major factors: static vs dynamic conditions and inter- vs intraindividual variability of the controlled or regulated variables.

\section{Conflict of Interest: None disclosed.}

2. Funding: This review received no specific funding.

\section{Tuesday, May 14, 2013}

$\mathrm{T} 4: \mathrm{PL}$

\section{Judging Nudging: Can nudging change behaviour to improve health?}

\section{Marteau T}

Behaviour and Health Research Unit, University of Cambridge, United Kingdom

The idea that behaviour can be changed by nudging people - through changing the environments within which their behaviour occurs received considerable impetus from the publication of the book 'Nudge: Improving decisions about health, wealth and happiness' in 2008 . By focusing upon interventions that do not require conscious engagement, i.e. that work via automatic processes, nudging could be more effective at changing behaviour than interventions requiring conscious engagement, particularly for those who are more socially deprived. Despite great appeal to policy makers, empirical evidence to support these effects is limited.

This talk will draw upon the results of a large scale scoping review and laboratory based experiments to estimate the effectiveness of nudging at changing behaviour to prevent obesity and reduce the social patterning associated with it.

T3:PL

\section{Strategy for Europe on Nutrition, Overweight and Obesity-related Health issues}

\section{Roux $P$, Kjaer GM}

European Commission, Belgium

Introduction: More than half $(52 \%)$ of the adult population in the European Union are overweight or obese with the rate of obesity having more than doubled over the past 20 years in most EU countries for which data are available.

Methods: Since 2007, the Commission has promoted EU action to enhance physical activity levels and promote healthier diets as set out in the Strategy for Europe on Nutrition, Overweight and Obesity-related Health issues. This strategy has as its main purpose to contribute to the reduction of the risks associated with poor nutrition and limited physical activity in the European Union. The Strategy calls for action in six priority areas: better informed consumers; making the healthy option available; encouraging physical activity; develop the evidence base to support policy making; develop monitoring systems, and putting children and low socio-economic groups as priority. One of the main tools of the European Strategy is developing Effective Partnerships. This has been put in place through the High Level Group for Nutrition and Physical Activity, and the Platform for action on Diet, Physical Activity and Health.

In the High Level Group the Commission works closely with Member States on issues such as food reformulation and in the EU Platform, the Commission encourages EU stakeholders to commit to concrete actions on diet and physical activity.

Results/Conclusion: At the moment, the Strategy on Nutrition, Overweight and Obesity-related Health issues is being evaluated and the final report is expected in April 2013. The outcome of the evaluation will serve as a valuable source for possible new actions. 
T3:PL

\section{Obesity prevention across Europe: Towards an integrated approach}

\section{Brug J}

Director EMGO Institute for Health and Care Research, Chair of Division VI of the VU University Medical Center, Professor of Epidemiology, VU University Medical Center, Amsterdam, the Netherlands.

The worldwide prevalence of overweight and obesity has been steadily increasing, also among children and adolescents, and has reached alarming epidemic proportions in Europe and elsewhere. Childhood overweight is a main predictor of adult overweight and obesity. Obesity treatment is largely ineffective, and a still stronger focus on prevention is needed. Such prevention should take a population and a life course approach and should focus on promoting healthy energy balance-related behaviors (EBRB) rather than weight management or weight loss.

EBRBs, i.e. healthy physical activity and eating as well as fewer sedentary activities, are a result of personal motivations and abilities, and environmental opportunities. It is not personal determinants or environmental opportunities that make a difference; it is mediation and interaction between and across these categories of behavioral determinants that drive healthful behaviors. To effectively encourage and facilitate healthful EBRBs health promotion efforts - to improve motivation and abilities- as well as health protection efforts - to ensure an 'obesopreventive' environment- are needed. Such an integrated - systemsapproach needs to be supported by participation of the main stakeholders in all phases of intervention and policy development, implementation and evaluation. Such integrated prevention approaches have and are being developed and testes across Europe. In this presentation, data results, ongoing efforts and new plans for cross-European research such as the European Commission funded HOPE (Health promotion through Obesity Prevention across Europe), ENERGY (EuropeaN Energy-balance Research to prevent excessive weight Gain among Youth), and SPOTLIGHT (Sustainable prevention of obesity through integrated strategies) projects will be presented.

\section{Wednesday, May 15, 2013}

\section{T5:PL}

\section{The obesity paradox: When is weight loss bad for you?}

Oppert, JM

Dept of Nutrition, Pitie-Salpetriere Hospital; Pierre et Marie Curie University-Paris6 ; Institute of Cardiometabolism and Nutrition (ICAN), Paris, France

While it is generally accepted that being obese carries risks to health and decreases life expectancy, there is still disagreement as to whether weight loss produces benefits on mortality and major health outcomes, especially cardiovascular events. The term 'obesity paradox' originates from the fact that obesity is a risk factor for heart failure but appears as a positive predictor for survival after the onset of heart failure. This concept provides a starting point to discuss the potential benefits of increased body fat and the potential negative consequences of weight loss. The evidence from observational studies appears mixed and intervention studies with hard endpoints are few. Although some observational studies have shown weight loss to be associated with increased mortality, there are major issues that would need to be taken into account. This includes a delineation between cardiovascular and other outcomes, age effects, the distinction between intentional and unintentional weight loss, the magnitude of weight loss (moderate as induced by behavioural change vs. massive loss through bariatric surgery), the composition of weight loss (the differential health effects of body fat and lean mass loss), the location of fat loss (the putative beneficial effects of gluteofemoral fat as opposed to abdominal visceral fat). The role of physical activity may be critical to modulate risks associated with weight loss through its influence on fitness and body composition but needs to be further assessed. The discussion brings to the issue of re-defining what success means in terms of a comprehensive approach to weight management.

1. Conflict of Interest: none.

2. Funding: Supported in part by the French National Research Agency (ANR), the French National Cancer Institute (INCa). 